\title{
Stochastic transitions between attractors in a tristable thermochemical system: competition between stable states
}

\author{
Bogdan Nowakowski ${ }^{1,2}$ - Andrzej L. Kawczyński
}

Received: 24 August 2017/Accepted: 21 October 2017/Published online: 7 November 2017

(C) The Author(s) 2017. This article is an open access publication

\begin{abstract}
We present the thermochemical model in the tristable regime which is characterized by three distinct attractors: two stationary states and a limit cycle. Transitions between the attractors are studied on the basis of the stochastic dynamics at the mesoscopic level, in which internal fluctuations are described by means of the master equation. Simulations of the stochastic dynamics follow the master equation, which has the specific form due to the necessary description of a continuous spectrum of temperature changes related to the exchange of energy with a thermostat. The mean first passage times are calculated for transitions from the limit cycle to each stable steady state. The effect of competition between the states is demonstrated: the duration of the passage can be strongly increased if the system during the transition to one state is also visiting the second competitive one. The variation of the passage times is studied for systems with different reaction heat, and the obtained dependence is explained by the location of the attractors and repellers in the phase space of system temperature and composition. The proportion between the mean passage times for the two states can be related to their relative strength of attraction.
\end{abstract}

Keywords Transitions in multistable system - Tristable thermochemical system · Master equation for thermochemical system · Competition between steady states

Electronic supplementary material The online version of this article (http://doi.org/10.1007/s11144017-1294-8) contains supplementary material, which is available to authorized users.

Bogdan Nowakowski

bnowakowski@ichf.edu.pl

1 Physics Laboratory, Warsaw University of Life Sciences, Nowoursynowska 159, 02-776 Warsaw, Poland

2 Institute of Physical Chemistry, Polish Academy of Sciences, Kasprzaka 44/52, 01-224 Warsaw, Poland 


\section{Introduction}

Fluctuations in nonequilibrium chemical systems, governed by nonlinear dynamics, may qualitatively change evolution in comparison to predictions provided by deterministic description [1, 2]. Despite the usually accepted scaling of fluctuations as small in magnitude, the induced effects can easily reach the macroscopic level. These prominent changes appear in far-from-equilibrium systems, close to bifurcations leading to new dynamical regimes specific for nonequilibrium conditions, like excitability, simple and complex oscillations, and multistability. The BelousovZhabotinsky [3, 4] and Bray-Liebhavsky [5, 6] reactions are the best known examples of chemical systems exhibiting a rich variety of nonlinear phenomena. Fluctuations also attract considerable attention since their positive, constructive role has been revealed in the phenomena of stochastic and coherence resonances [7-10], analyzed theoretically $[11,12]$ and observed in experiments $[13,14]$ as well.

Particularly expressive effects of fluctuations can be expected in multistable systems which have a few attractors for dynamics, possibly even of different types. Under such conditions, fluctuations can trigger the system between different attractors, leading to qualitative, measurable changes in macroscopic variables. In the present work, we study the transitions theoretically, applying simulations of stochastic dynamics described at the mesoscopic level by the master equation. We use the convenient, previously developed model of thermochemical systems [15-18], which can be described deterministically by two equations only, but nevertheless exhibits all features of nonlinear dynamics. The model allowed us to obtain results for coherence resonance in excitable systems [17-19], stochastic resonance in oscillating systems [19], and probability distribution for transitions in bistable systems with limit cycles $[15,16]$. In this work, we give the setup of the tristable regime, in which the model has three attractors: the limit cycle and two stationary states. We then study transitions in detail beginning from the cycle and terminated on the steady states. In the next section, we describe the thermochemical model and its deterministic and stochastic dynamics. The results are presented in the third section: first the construction of the tristable regime, and then the mean passage times obtained from the stochastic simulations.

\section{Thermochemical model}

We model a reactor which contains a homogeneous dilute mixture of three gases $(A$, $B$ and $C$ ) and is in thermal contact with a surrounding thermostat maintained at constant temperature $T_{b}$. The catalyst $C$ transforms the reactant $A$ into the product $B$ in the bulk reaction:

$$
A+C \stackrel{k_{1}}{\rightarrow} B+C+Q
$$

$Q$ denotes a reaction heat released in exothermic Reaction 1. The second process mimics the inflow of the substrate and removal of the product and maintains the system out of thermodynamic equilibrium: 


$$
B \stackrel{k_{2}}{\rightarrow} A
$$

We assume this is a surface reaction proceeding without activation energy on the boundaries of the reactor. Reactions 1-2 do not change the total population $N$ of particles and the catalyst population $N_{\mathrm{C}}$, so the chemical composition of the mixture is determined by the concentration of single species, $A$ or $B$. The corresponding constant concentration $n-n_{\mathrm{C}}=n_{\mathrm{A}}+n_{\mathrm{B}}$ can be used as a convenient scaling factor. The internal energy $U$ of the dilute gas system can be expressed by the temperature $T$ with the use of the relation: $U=(3 / 2) N k_{\mathrm{B}} T$, where $k_{\mathrm{B}}$ is the Boltzmann constant. The heat flow between the reactor and the thermostat is proportional to their temperature difference, according to the Newton's law. The reaction rate constants and the rate of heat exchange follow the kinetic theory result for collision frequency, which gives the square root dependence on temperature, $T^{1 / 2} ; k_{1}$ includes in addition the Arrhenius factor $\exp \left(-E / k_{\mathrm{B}} T\right)$, where $E$ is an activation energy for Reaction 1.

The deterministic dynamics of the system is described by two equations only [17, 18], for dimensionless concentration of $A$ species $\alpha=n_{A} /\left(n_{A}+n_{B}\right)$, which determines the composition, and reduced temperature $\theta=T / T_{b}$ which determines energy:

$$
\begin{aligned}
& \frac{\mathrm{d} \alpha}{\mathrm{d} t}=\sqrt{\theta}\left[-\alpha \exp (-\varepsilon / \theta)+K_{2}(1-\alpha)\right] \\
& \frac{\mathrm{d} \theta}{\mathrm{d} t}=\frac{2}{3} \sqrt{\theta} q\left[\alpha \exp (-\varepsilon / \theta)-K_{1}(\theta-1)\right]
\end{aligned}
$$

Time in Eqs. 3 and 4 is $t=n_{\mathrm{C}} k_{1}^{0} t_{1}$, where $t_{1}$ is a real time and rate constant $k_{1}^{0}$ is extracted of temperature dependence, $\varepsilon=E / k_{\mathrm{B}} T_{b}, \quad q=Q\left(n-n_{C}\right) / n k_{B} T_{b}$. The dimensionless constants $K_{1}=\kappa^{0} n S / q k_{1}^{0} n_{C}\left(n-n_{C}\right) V$ and $K_{2}=k_{2}^{0} S / k_{1}^{0} n_{C} V$ involve temperature-independent heat transfer coefficient $\kappa^{0}$ and rate constant $k_{2}^{0}$, and the system volume $V$ and surface $S$. In Eq. 4, $q$ is the scaling factor which controls the speed of dynamics for $\theta$. The steady states of the system are determined by intersections of the nullclines for Eqs. 3 and 4, respectively given by:

$$
\begin{gathered}
\alpha_{A}=1 /\left[1+\left(1 / K_{2}\right) \exp (-\varepsilon / \theta)\right] \\
\alpha_{T}=K_{1}(\theta-1) \exp (\varepsilon / \theta)
\end{gathered}
$$

The analysis of the dynamics reported in the previous papers [15-18] has shown that nonlinear effects can be observed only for $\varepsilon>4$, when the nullcline for temperature is a non-monotonic, $\mathrm{N}$-shaped function of $\theta$. The middle branch of $\alpha_{T}$ lying between the extremes has a negative slope and is repelling. As $q$ is the control parameter for strength of dynamics for $\theta$, with increasing $q$ the unstable middle branch of $\alpha_{T}$ becomes more repulsive, so the stability of steady states on it is 
weakening. The system can have at most three stationary states, and only two of them can be stable leading then to bistability.

The stochastic description is based on the function $P\left(\theta, N_{A}, t\right)$, which gives the probability distribution that the system is in a state $\left(\theta, N_{A}\right)$. The population of particles $N_{\mathrm{A}}$ is an equivalent variable to the deterministic fraction concentration $\alpha=N_{A} / N$, but is here more convenient and exact, since jumps $\Delta N_{A}$ due to the reactions are fixed. The dynamics of $P$ follow the master equation $[17,18,20]$ :

$$
\begin{aligned}
\frac{\partial}{\partial t} P\left(\theta, N_{A}, t\right)= & \sum_{\Delta N_{A}} \int_{\Delta \theta<\theta} d(\Delta \theta) P\left(\theta-\Delta \theta, N_{A}-\Delta N_{A}, t\right) \\
& \times w\left(\theta-\Delta \theta, N_{A}-\Delta N_{A} \rightarrow \theta, N_{A}\right) \\
& -P\left(\theta, N_{A}, t\right) \sum_{\Delta N_{A}} \int_{\Delta \theta>-\theta} d(\Delta \theta) w\left(\theta, N_{A} \rightarrow \theta+\Delta \theta, N_{A}+\Delta N_{A}\right)
\end{aligned}
$$

Transition function $w$ gives rates of transition from a state $\left(\theta, N_{A}\right)$ to $\left(\theta+\Delta \theta, N_{A}+\Delta N_{A}\right)$; it is a discrete function of $N_{A}$, but a continuous one for $\theta$ due to a continuous spectrum of jumps $\Delta \theta$ related to stochastic exchange of energy with the thermostat. The explicit form for function $w$ is given in the online Appendix.

We do not try any solution of complicated integro-differential equation in Eq. 7. Instead, we use the kinetic Monte Carlo simulation method which provides stochastic trajectories evolving according to the master equation. The Monte Carlo method is well known in the case of the master equation for discrete jumps of species populations in chemical systems without thermal effects [21, 22], and was applied also in kinetics of biological processes [23]. In the previous works [18, 20], we presented extension of this approach for the master equation in the integral form (7).

We follow the general rules for the simulation algorithm of trajectory which consists of a sequence of steps, entirely determined by waiting times $\Delta t$ and coordinates $\left(\Delta \theta, \Delta N_{A}\right)$ of consecutive jumps. For a step beginning at time $t$ in $\left(\theta, N_{A}\right)$, the total rate of escape from this state is calculated by summing particular rates of all possible transitions, $W_{\text {tot }}\left(\theta, N_{A}\right)=\sum_{\Delta N_{A}} \int_{\Delta \theta>-\theta} d(\Delta \theta) w\left(\theta, N_{A} \rightarrow \theta+\Delta \theta, N_{A}+\right.$ $\left.\Delta N_{A}\right)$, and then the waiting time $\Delta t$ for the passage is chosen from the exponential distribution with the mean $1 / W_{\text {tot }}$. The jump is selected according to its contribution to the transition function, i.e. the increment $\left(\Delta \theta, \Delta N_{A}\right)$ is sampled from the normalized probability distribution $J\left(\Delta \theta, \Delta N_{A}\right)=w\left(\theta, N_{A} \rightarrow \theta+\Delta \theta, N_{A}+\Delta N_{A}\right) / W_{\text {tot }}\left(\theta, N_{A}\right)$. The state of the system is then updated to $\left(\theta+\Delta \theta, N_{A}+\Delta N_{A}\right)$ at time $t+\Delta t$, a next step is generated and so on. The details have been reported previously [18, 20]. 


\section{Results}

\section{Setup of tristable system}

We have previously demonstrated that the bistable regime can be obtained in the frame of our thermochemical model [15-18]. However, it is much more difficult task to find a tristable system. Constructing the system with three attractors one has to realize that only two of them can be stationary states, because the nullclines $(5,6)$ can be have at most three intersection points, one of which must be then a saddle point. Another important indication follows from the stability analysis (which can be based on linear dynamics), according to which a stationary state located on the repulsive branch of $\alpha_{T}$ ultimately becomes unstable for sufficiently high $q$. Thereafter, the only attractor left with $q$ increasing is the limit cycle surrounding all three states, which appears after the homoclinic-orbit bifurcation [15].

Thus, our aim is to find the range of $q$, for which the limit cycle is formed already and it coexists with the still stable two steady states, before they lose stability with increasing $q$. For the chosen values $\varepsilon=4.9$ for the activation energy and $K_{1}=0.07128862, K_{2}=0.5489482$ for the rate constants, the system has three

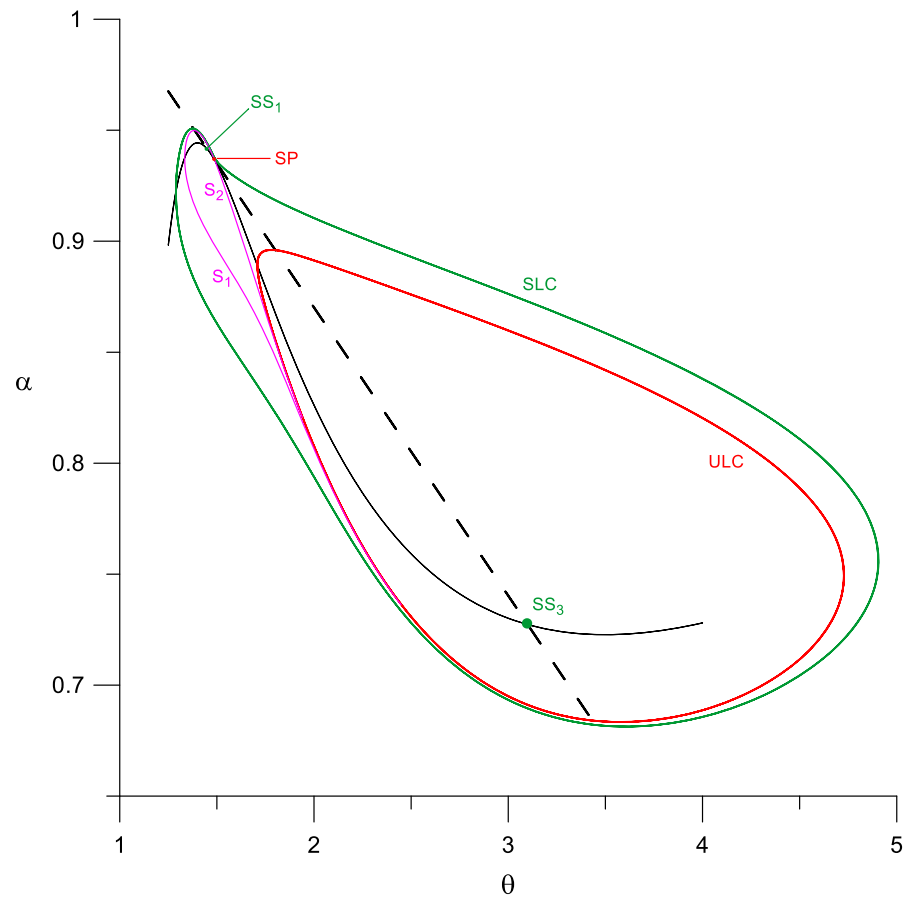

Fig. 1 Phase portrait of the tristable system on plane $(\theta, \alpha)$ for the following values of the parameters $\varepsilon=4.9, K_{1}=0.07128862$ and $K_{2}=0.5489482$ at $q=160$. Nullclines (in black): $\alpha_{T}$ for $\theta$ (solid line) and combination of right sides of Eqs. $(3,4)(\alpha-1)=-(\theta-1) K_{1} / K_{2}$ (dashed line). Attractors (in green): steady states $\mathrm{SS}_{1}$ and $\mathrm{SS}_{3}$, and the stable limit cycle (SLC). Repellers (in red): the saddle point (SP) and the unstable limit cycle (ULC). Separatrices (in magenta) $S_{1}$ and $S_{2}$ entering to SP and separating the basin of attraction of $\mathrm{SS}_{1}$ from the basin of attraction of SLC. (Color figure online) 


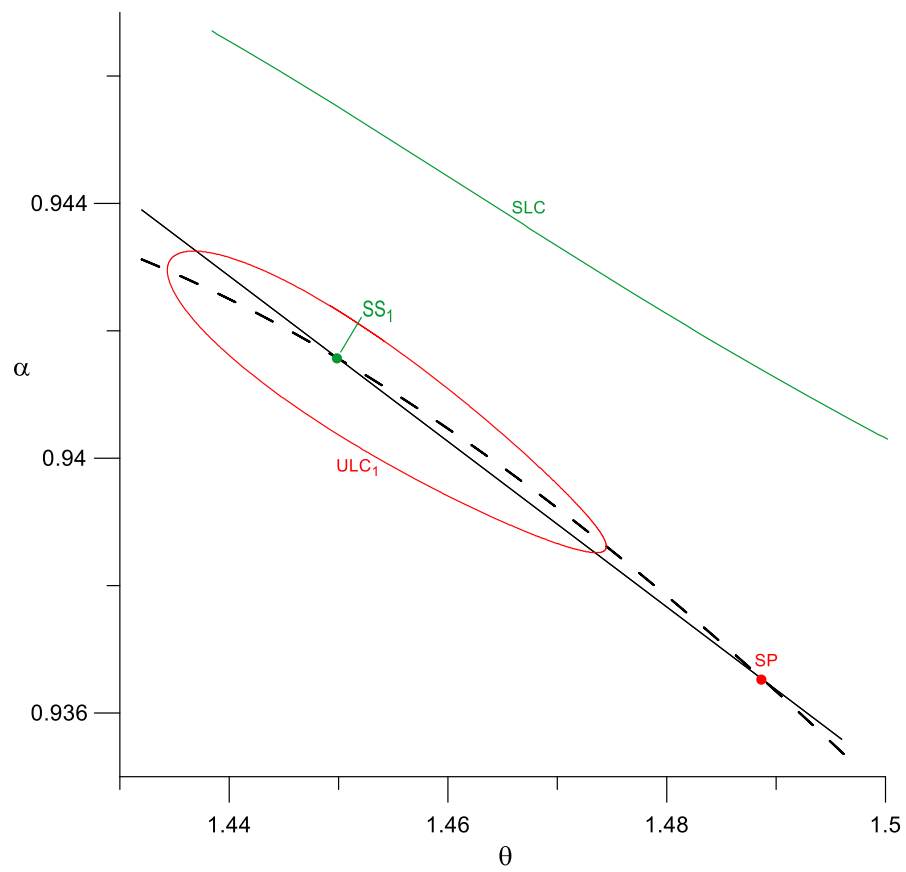

Fig. 2 Enlargement of fragment of phase portrait showing the neighborhood of stable state $\mathrm{SS}_{1}$ and saddle point SP at $q=240$. Unstable limit cycle ( $\left.\mathrm{ULC}_{1}-\mathrm{red}\right)$ surrounding $\mathrm{SS}_{1}$ limits basin of attraction. Fragment of large stable limit cycle (SLC_-green). (Color figure online)

stationary states on the repulsive branch. Two of them: the low-temperature steady state $\mathrm{SS}_{1}$ given by the intersection point in the phase plane $(\theta, \alpha)$ at $(1.45$, $0.941561191)$, and the high-temperature steady state $\mathrm{SS}_{3}$ at $(3.1,0.727285558)$ are nodes or focuses and are stable for sufficiently low $q$. The middle one at $(1.4888988,0.936509634)$ is always a saddle point SP. The phase portrait of the system is presented in Fig. 1. The calculation of the deterministic dynamics shows that at $q=157.02$ the homoclinic-orbit bifurcation yields the large stable limit cycle surrounding the two steady states which are still stable. The basin of attraction of the high-temperature stable steady state is bounded by the unstable limit cycle surrounding $\mathrm{SS}_{3}$. As $q$ increases, the unstable cycle tightens around $\mathrm{SS}_{3}$ and at $q=224.446$ it absorbs the steady state which loses stability after the reverse Hopf bifurcation. The low-temperature stable steady state is very close to the saddle point, and its basin of attraction is originally limited by the separatrices which spin out from the unstable cycle and enter to the saddle. The stability evolution for $\mathrm{SS}_{1}$ is governed by the similar scenario: Increasing of $q$ leads at $q=231.605$ to contact between separatrix $S_{1}$ and the separatrix entering to $S_{1}$, which yields the homoclinic-orbit bifurcation and birth of an unstable limit cycle. $\mathrm{SS}_{1}$ and the cycle formed around it are very close to the saddle point, and the corresponding part of the phase portrait is magnified in Fig. 2 (for $q=240$ ) in order to show the details. The unstable cycle clamps around $\mathrm{SS}_{1}$ as $q$ increases, and eventually the low- 
temperature stable steady state becomes unstable for $q=251.635$. To sum up, in the range $157.02<q<224.446$ we obtain the tristable system in which the two stable steady states (focuses) are surrounded by the stable limit cycle.

\section{Results of stochastic simulations}

Simulations of the stochastic dynamics of the thermochemical system are performed by means of the kinetic Monte Carlo method according to the developed algorithm based on the master equation given in Eq. 7. We study the described above system in the range $160<q<220$, where it is in the tristable regime. A stochastic trajectory is initiated on the large stable limit cycle, and during its evolution we observe transition back and forth to the interior stable stationary states $\mathrm{SS}_{1}$ and $\mathrm{SS}_{3}$, induced by internal, intrinsic fluctuations. We calculate the first passage times from the limit cycle to the steady states.

The transitions from the stable limit cycle to the steady state $\mathrm{SS}_{1}$ can process in two different manners. The first way is straightforward, it means the trajectory jumps from the cycle directly to $\mathrm{SS}_{1}$ after crossing the separatrix $\mathrm{S}_{1}$ entering the saddle point and bounding the basin of attraction of the stable state. The second possible type of transition consists of two phases: (1) First the trajectory leaves the stable cycle by jumping over the unstable limit cycle that limits the basin of attraction of the stable state $\mathrm{SS}_{3}$, and when inside the unstable cycle it descends and spends some time around $\mathrm{SS}_{3}$; (2) Then, after some lapse of time, it leaves this basin of attraction and in course of its further evolution it finally reaches the stable steady state $\mathrm{SS}_{1}$. During this second phase of transition it can pass either along the inward side of the separatrix $S_{1}$, or even it can return to the stable cycle and begin once again a transition to $\mathrm{SS}_{1}$ (in any possible manner).

Fig. 3 shows the mean first passage times $\tau_{\mathrm{i}}$ to $\mathrm{SS}_{1}$ for systems with various reaction heat $160<q<220$, for the indirect transitions, i.e. when during the transition from the stable limit cycle the system visits first the stable steady state $\mathrm{SS}_{3}$ before eventually it reaches the steady state $\mathrm{SS}_{1}$. The times $\tau$ can be compared with the period of the stable limit cycle, which is $T=27.43$ for $q=160$. The passage time decreases as a function of $q$. There are a few factors which results in such dependence. Let us notice that the passage time is particularly high for the lowest value of $q$. In this case, the system is very close to the homoclinic orbit bifurcation leading to the appearance of the stable limit cycle, and in such system the stable cycle and the unstable one around $\mathrm{SS}_{3}$ are very close each other. Consequently, the passage from the stable cycle over the unstable one is relatively more probable, and then the system enters into the basin of attraction of $\mathrm{SS}_{3}$. For low values of $q$, this state is more stable because its attraction domain is more extended, limited by bigger unstable limit cycle. The system spends a long time period in a vicinity of $\mathrm{SS}_{3}$ before it leaves this area and can continue transition to $\mathrm{SS}_{1}$. This long residence time around $\mathrm{SS}_{3}$ gives contribution to passage time $\tau_{\mathrm{i}}$, which is the larger the stronger is $\mathrm{SS}_{3}$, which is for lower values of $q$. As $q$ increases, the unstable limit cycle around $\mathrm{SS}_{3}$ decreases and $\mathrm{SS}_{3}$ becomes weaker. Then both the probability of catching the system towards $\mathrm{SS}_{3}$ and the duration of residence around this state are decreasing, and these two factors consistently lead to 


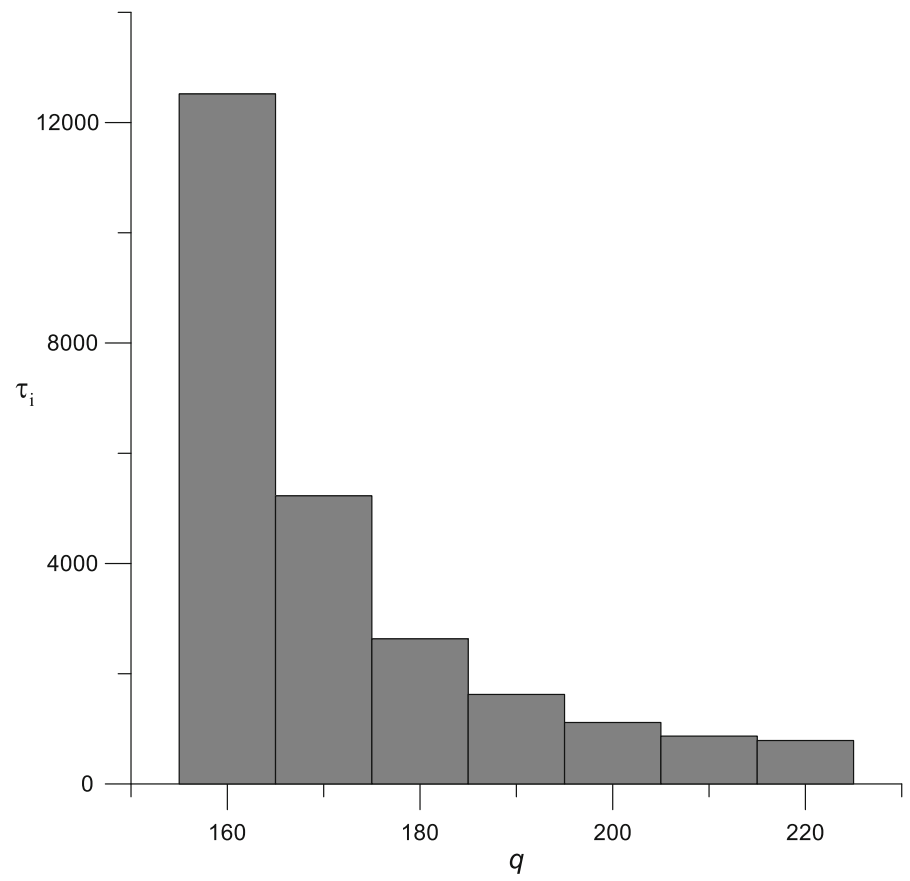

Fig. 3 Mean first passage times from large stable limit cycle to stable state $\mathrm{SS}_{1}$ for indirect transitions (with intermediate visit to $\mathrm{SS}_{3}$ ) for systems with different reaction heat $q$

shortening of transition times to $\mathrm{SS}_{1}$. It should also be noticed that for higher $q$, the evolution of the system in general becomes faster because $q$ is a scaling factor in Eq. 4 for the dynamics of temperature. For example, due to this speed-up, the period for the stable limit cycle decreases to $T=14.48$ for $q=220$, compared to the above given value of $T$ for $q=160$. Fig. 4 shows the mean first passage times $\tau_{\mathrm{d}}$ to $\mathrm{SS}_{1}$ as a function of $q$ during direct transitions, without visiting the steady state $\mathrm{SS}_{3}$. In this case, the passage times $\tau_{\mathrm{d}}$ shows a weak dependence for initial low values of $q$, and then distinctly increases for systems with higher reaction heat. Such dependence follows from the opposite effects of two factors. As $q$ increases, the system approaches bifurcation in which $\mathrm{SS}_{1}$ loses stability. The stability of $\mathrm{SS}_{1}$ is weakening and the associated decrease of strength of its attraction leads to longer transition times to this state. On the other hand, the dynamics of the system becomes faster for higher $q$, and consequently this feature has the effect of shortening of passage times. Initially, for lower values of $q$, the two effects are comparable, giving the flat dependence of $\tau_{\mathrm{d}}$ on $q$, but as $q$ increases the first factor evidently prevails and results in longer mean passage times.

Analyzing the transitions from the stable limit cycle to the stable steady state $\mathrm{SS}_{3}$, we similarly find either direct passages of the system from the cycle to $\mathrm{SS}_{3}$ without visiting $\mathrm{SS}_{1}$, or indirect ones when the system in a course of transition is catch by the second state $\mathrm{SS}_{1}$ and stays for some time around it before it leaves the basin of attraction of $\mathrm{SS}_{1}$ and evolves further towards $\mathrm{SS}_{3}$. To reach state $\mathrm{SS}_{3}$, the system 


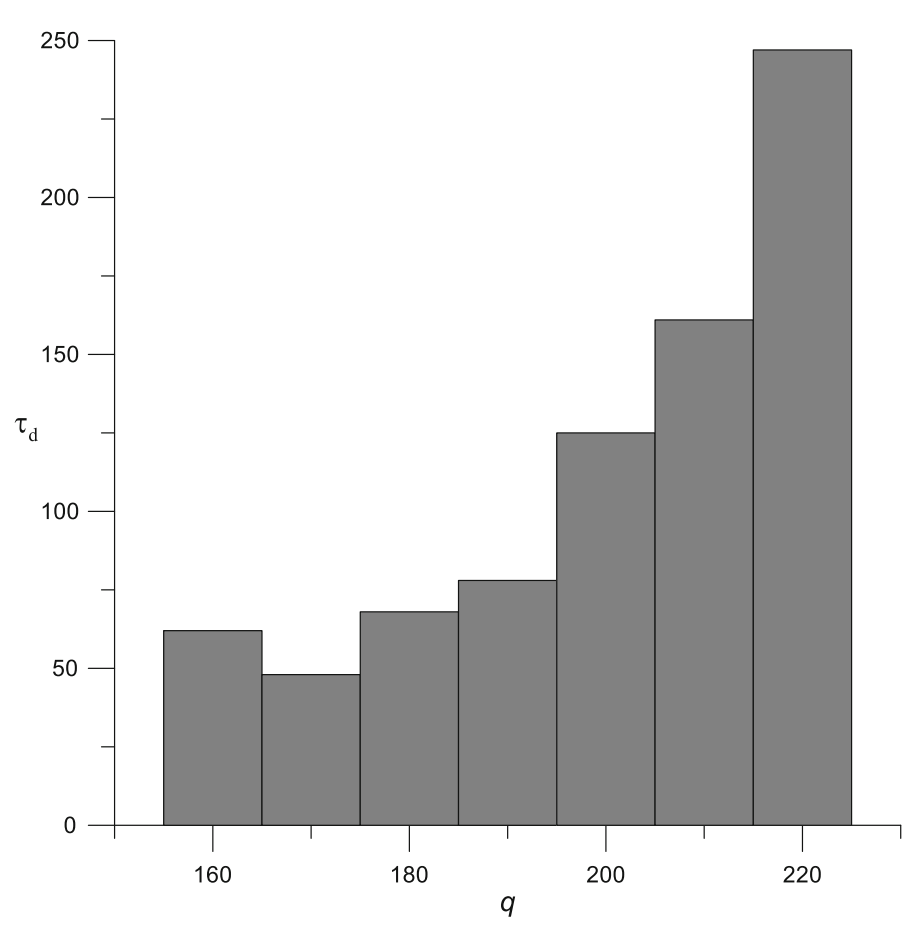

Fig. 4 Mean first passage times for direct transitions from large stable limit cycle to stable state $\mathrm{SS}_{1}$ for systems with different reaction heat $q$

must pass the surrounding $\mathrm{SS}_{3}$ unstable limit cycle which is a boundary for the basin of attraction around $\mathrm{SS}_{3}$ and corresponds to a repelling barrier which has to be overcome in a course of transition from the stable cycle. For lower values of $q$ that is closer to bifurcation, which yields the stable cycle, both cycles remain close to each other in a large region of the phase space, and in this case the barrier is relatively narrower and weaker. Consequently, the passage over the unstable cycle becomes more probable, and the mean passage times for systems in this range of $q$ are relatively shorter. If $q$ grows, the unstable limit cycle decreases and tightens around $\mathrm{SS}_{3}$, then the distance in the phase plane between the two cycles becomes larger. The barrier formed by the unstable cycle is accordingly wider and higher, the probability of transition decreases and the mean first passage times increases.

Fig. 5 presents the mean first passage times $\tau$ for transitions from the stable limit cycle to the stable state $\mathrm{SS}_{3}$, as a function of reaction heat $q$. The expected, explained above increase of $\tau$ or growing $q$ is confirmed, indicating that the magnitude of the barrier is the most significant factor. Evidently, the acceleration of dynamics for higher $q$, which leads to shortening of passage times, is the less important secondary factor in this case. It can be reminded that the calculation of mean first passage time is possible for a one-variable stochastic system [1, 24]. The analytical result derived from the Fokker-Planck equation shows the exponential dependence on the magnitude of the barrier related to unstable state separating attractors [24]. Although the analytical solution cannot be generalized for two- 


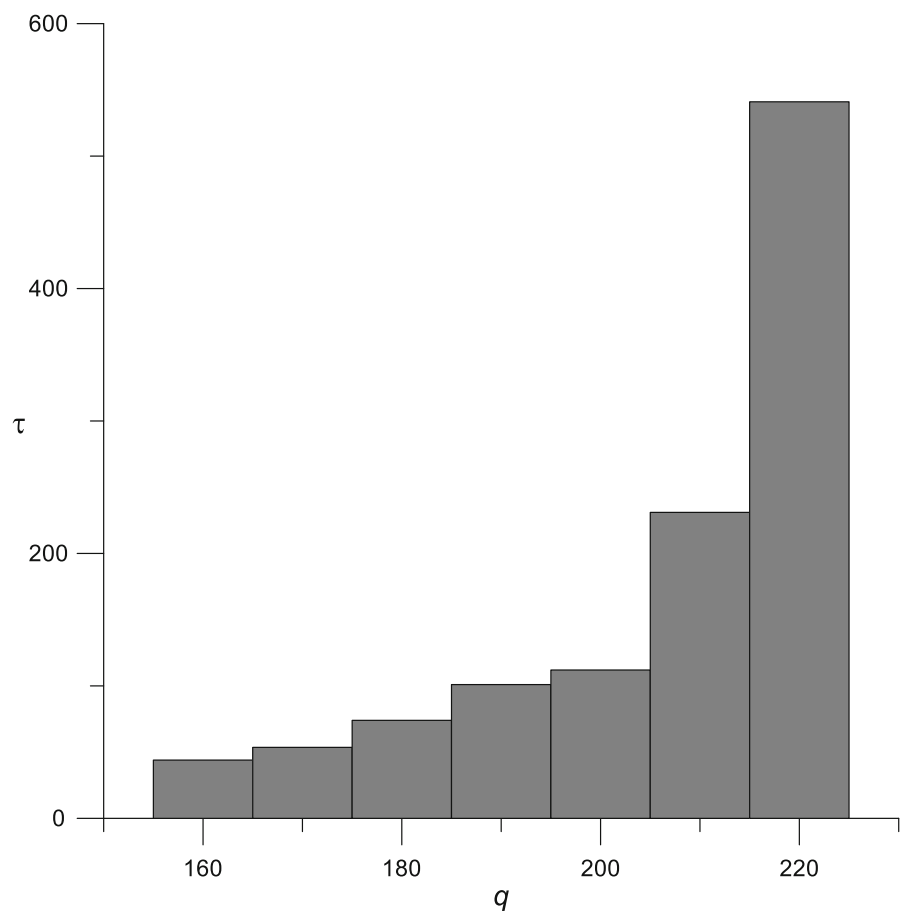

Fig. 5 Mean first passage times from large stable limit cycle to stable state $\mathrm{SS}_{3}$ for systems with different reaction heat $q$

variable system, this type of strong dependence should remain at least qualitatively valid.

The values of the obtained mean passage times in the range of lower $q$ are shorter for transitions to $\mathrm{SS}_{3}$, while $\tau$ for $\mathrm{SS}_{1}$ is shorter for higher $q$. The studied system belongs to the class of non-conservative multivariable systems for which it is not possible to introduce in dynamics a potential and use its depth as a precise measure of stability of the stationary states. However, a relation of the mean first passages time for various attractors can give us an idea about their relative attraction strength: more effective attraction should result in faster evolution towards stronger stable states. From this point of view, the obtained results indicate that the stronger steady state (relative to transitions from the stable limit cycle) is $\mathrm{SS}_{3}$ for low $q$ and $\mathrm{SS}_{1}$ when $q$ is higher. Weakening of $\mathrm{SS}_{3}$ stability as $q$ increases is related to decline of the unstable limit cycle which limits the decreasing basin of attraction for $\mathrm{SS}_{3}$.

\section{Summary}

We have shown that the model of thermochemical system described only by two variables can exhibit nevertheless rich dynamical behavior including multistability, in which different types of attractors coexist. It can be noticed, that the model 
includes at most bimolecular reactions and this feature makes it more suitable for simulations at the molecular level [24, 25].

Tuning the parameters, we have found values which provide tristable system with two stable steady states surrounded by the stable limit cycle. The simulations of the stochastic dynamics in the tristable regime reveal transitions among attractors. We have studied in detail passages from the limit cycle to each of the steady states. These transitions can be either direct or indirect when in a course of passage the system visits the other steady state. The mean passage time for indirect transitions can be much longer than for direct ones, if the second state, intercepting the trajectory, is well stable. The relation between the values of the mean passage times to the two steady states can provide an indication which state exhibits higher stability.

Open Access This article is distributed under the terms of the Creative Commons Attribution 4.0 International License (http://creativecommons.org/licenses/by/4.0/), which permits unrestricted use, distribution, and reproduction in any medium, provided you give appropriate credit to the original author(s) and the source, provide a link to the Creative Commons license, and indicate if changes were made.

\section{References}

1. van Kampen NG (1987) Stochastic processes in physics and chemistry. North-Holland, Amsterdam

2. Nicolis G, Prigogine I (1977) Self-organization in nonequilibrium systems. Wiley, New York

3. Zhabotinsky AM (1974) Concentration oscillations. Nauka, Moskwa (in Russian)

4. Zhabotinsky AM (1991) Chaos 1:379

5. Schmitz G, Kolar-Anić L, Anić SR, Čupić ŽD (2008) J Phys Chem A 112:13452-13457

6. Vukojević V, Anić S, Kolar-Anić L (2000) J Phys Chem A 104:10731-10739

7. Nicolis C (1981) Sol Phys 74:473-478

8. Benzi R, Sutera A, Vulpiani A (1981) J Phys A 14:L453-L457

9. Gammaitoni L, Hänggi P, Jung P, Marchesoni F (2009) Eur Phys J B 69:1

10. Lindner B, Garcia-Ojalvo J, Neiman A, Schimansky-Geier L (2004) Phys Rep 392:321

11. Anischenko A, Astakhov V, Neiman A, Vadivasova T, Schimansky-Geier L (2002) Nonlinear dynamics of chaotic and stochastic systems. Springer, Berlin

12. Sagues F, Sancho JM, Garcia-Ojalvo J (2007) Rev Mod Phys 79:829

13. Alcor D, Croquette V, Jullien L, Lemarchand A (2004) Proc Natl Acad Sci USA 101:8276

14. Faisal AA, Selen LP, Wolpert DM (2008) Nat Rev Neurosci 9:292

15. Nowakowski B, Kawczyński AL (2005) J Phys Chem A 109:3134

16. Nowakowski B, Kawczyński AL (2006) ChemPhysChem 7:502

17. Kolbus A, Lemarchand A, Kawczyński AL, Nowakowski B (2010) Phys Chem Chem Phys 12:13224

18. Nowakowski B, Kawczyński AL, Kolbus A, Lemarchand A (2011) Eur Phys J B 84:137-145

19. Lemarchand A, Gorecki J, Gorecki A, Nowakowski B (2014) Phys Rev E 89:022916

20. Nowakowski B, Lemarchand A (2001) Phys Rev E 64:061108

21. Gillespie DT (1976) J Comput Phys 22:403

22. Gillespie DT (1977) J Phys Chem 81:2340

23. Elf J, Ehrenberg M (2004) Syst Biol 1:230; ibid, PLoS Comp Biol 1:e2 (2005)

24. Lemarchand A, Nowakowski B (2007) J Phys 19:065130

25. Kawczyński AL, Gorecki J (1992) J Phys Chem 96:1061 